\title{
An Identical Inputs-Adaptive Filter for the Detection of Signal's Breakdown Points
}

\author{
Mohammed Siddig H. Mohammed', Kim Ki-Seong2* \\ ${ }^{1}$ Department of Nuclear Engineering, King Abdulaziz University, Jeddah, KSA \\ ${ }^{2}$ Department of Mechanical Design Engineering, Chonnam National University, Yeosu, Korea \\ Email: *sngkim@chonnam.ac.kr
}

How to cite this paper: Mohammed, M.S.H. and Ki-Seong, K. (2017) An Identical Inputs-Adaptive Filter for the Detection of Signal's Breakdown Points. World Journal of Engineering and Technology, 5, 232-240.

https://doi.org/10.4236/wjet.2017.52018

Received: March 2, 2017

Accepted: May 8, 2017

Published: May 11, 2017

Copyright $\odot 2017$ by authors and Scientific Research Publishing Inc. This work is licensed under the Creative Commons Attribution International License (CC BY 4.0).

http://creativecommons.org/licenses/by/4.0/

\begin{abstract}
Detection of the signal's breakdown points is important for many science and engineering applications. Numerous signal processing methods have been used for this purpose. Of these, the adaptive prediction is simple and easy to implement, however; its simplicity and robustness are hindered by the required delay in the input signal. This paper introduces an efficient alternative to the adaptive prediction in the application of breakdown and inflection points' detection. Unlike the adaptive predictor, the proposed filter doesn't require a delay in the primary input to produce the filter's reference input, which significantly improves the computation speed and overcome the problem of performance sensitivity to the delay value. The Normalized Least-Mean Squares algorithm was used to realize both the adaptive predictor and the proposed filter. The filters were implemented in LabVIEW system design software. The performances of the filters were studied using simulated signals and the simulation results were verified using an experimental signal. The simulation and experimental results showed that the proposed filter efficiently detects the signal breakdowns. Furthermore, the simplicity of the filter offered a significant improvement in the computation speed.
\end{abstract}

\section{Keywords}

Breakdown Point, Abrupt Change, Adaptive Prediction, Identical Inputs-Adaptive Filter

\section{Introduction}

Abrupt changes in signals are characterized by the random time appearance and extremely short duration. Detection of these challenging signal events is crucial for many science and engineering applications such as in electrical systems.

The literature review reveals numerous signal processing methods that have 
been used for abrupt change detection such as control charts, filtered derivative algorithms, and the cumulative sum (CUSUM) algorithm [1]. One of the advanced methods used for abrupt change detection is the wavelet transform, which is more powerful than the time domain analysis methods because it has a good localization capability in both time and frequency domains [2]. The analysis of the wavelet coefficients has provided an accurate detection of breakdown point of the transient signal in the electrical circuits [3]. The electrical power systems disturbances of sudden impulses, voltage dips, and oscillatory transient sinusoids have been localized and estimated using a method based on the Continuous Wavelet Transform (CWT) and Discrete Wavelet Transform (DWT) [4]. The DWT has been used within an approach to detect the onset time, duration, and dominant frequencies of electricity supply disturbances. The efficiency and accuracy of the method have been demonstrated by processing test signals contain different types of disturbances [5].

Nevertheless, the adaptive prediction outperformed the wavelet transform methods by its simplicity in computations and implementation. In adaptive prediction, the future values of a signal are estimated based on the past values. The adaptive predictor has two inputs: the primary input; it is also known as the desired output, and a reference input, which is a delayed trace of the primary input. The adaptive predictor calculates the difference between the primary input and the filtered reference input to produce an error signal (Figure 1). The coefficients are adjusted so that the error signal is minimized, then the adaptive filter can predict the future values of the signal [6]. The abrupt change or breakdown point location is indicated by the prediction error.

Based on adaptive prediction, the principle of adaptive line enhancer using the LMS and recursive LMS has been applied to the modeling of transient vibration signals in machinery operation. The success of the adaptive modeling method was demonstrated by monitoring the transient running-up process of healthy and faulty motors [7]. An adaptive filter based on Least-Mean Squares (LMS) and Recursive Least Squares (RLS) has been developed to extract spheric signal, which is event-based signals. The input signals to the filter undergo two delays, the first one is to produce primary input, which is also delayed to give the reference input. This arrangement put the developed filter under the adaptive prediction class [8]. Linear adaptive neuron (Adaline) has been applied to detect power quality events. The Adaline could detect most common power quality faults, however; it was sensitive to the input delay [9].

The adaptive predictor is a computational device that implements the delay by passing the values of the signal, from a previous iteration in a loop, through to the next iteration. This shift in the data points slows the computational speed. Moreover, the quality of the output is sensitive to the selection of the delay time. These limitations hinder the application of adaptive prediction in real-time measurements.

In this paper, a faster and simpler adaptive filter that doesn't need a delayed input, to detect breakdown points is proposed. 


\section{The Proposed Method}

The input signals and their configuration are crucial for determining the function and the application of the adaptive filters. To avoid the burden implied by the delayed input in the adaptive prediction, an intuitive realization of an adaptive filter is proposed, it takes the measured signal as the reference and primary inputs at the same time. This realization is explained as follows:

The measured signal can be modeled as:

$$
y_{e}=x_{e} * u+n
$$

where: $y_{e}$ is the measured signal, $x_{e}$ is the input signal, $u$ is the impulse response of the measurement system, $n$ is noise, and * denotes the convolution.

The subscript (e) in $y_{e}$ and $x_{e}$ indicates that the measured signal contains a time-event.

If $y_{e}$ is passed through a FIR filter with an adaptive algorithm, the output will be in the form of:

$$
y(n)=\sum_{i=0}^{N-1} h_{i}(n) y_{e}(n-i)
$$

where: $h_{i}(n) \equiv$ the filter coefficients, and $y(n)$ indicates the time-event in $y_{e}$ is smoothed by the FIR filter.

The coefficients $h_{0}(n), h_{1}(n), \cdots, h_{N-1}(n)$ are selected so that the difference (error):

$$
e(n)=d(n)-y(n)
$$

is minimized with respect to mean-square-error (MSE), where:

$e(n) \equiv$ the error signal, and

$d(n) \equiv$ the primary signal.

If the measured $y_{e}$ is taken as the filter's reference and primary input at the same time, then the error signal of Equation (3) can be rewritten using Equation (1) and Equation (2) as:

$$
e(n)=y_{e}-y
$$

The error signal between a signal with an event and a signal with no or smaller size event should have a large value (peak or valley) at the location of the event and ideally zero or close to zero elsewhere. The location of the event is determined by the onset of the peak or valley in the error signal. This intuitive approach of the proposed filter was named the Identical Inputs-Adaptive Filter (IAF). Using the measured signal as the reference and primary input at the same time will simplify the implementation of the filter, desensitizes the filter's performance to the delay value selection, shorten the computation time and greatly supports the real-time application.

In this paper, the Normalized Least-Mean Squares (NLMS) algorithm is used to implement both the adaptive predictor and the IAF. The NLMS is an improved variant of the LMS algorithm, it enables a time-varying step size that results in an enhanced convergence speed and better handling of time-varying 
signals. The realization and mathematical formulation and of LMS and NLMS are well described in the literature, e.g. [6] [10].

The NLMS update the filter coefficients as follows:

$$
w(n+1)=w(n)+\frac{\mu n}{\gamma+X^{\mathrm{T}}(n) X(n)} e(n) X(n)
$$

where $\mu n$ is variable step size, $X^{\mathrm{T}}(n)$ is the transpose of the input signal vector $X(n)$ and $\gamma$ is a parameter should be included, in order to avoid large step sizes when $X^{\mathrm{T}}(n) X(n)$ becomes small [6].

The adaptive predictor and the proposed IAF were implemented using LabVIEW system design software.

\section{Simulation Results}

The first signal used in the simulation study was a sine wave with a breakdown point, shown in Figure 2(a). Figure 2(b) and Figure 2(c) show the results (error signals) of breakdown point detection attempts by the adaptive linear prediction and the IAF, respectively. Both methods demonstrated a comparable convergence speed, moreover; they were equally able to accurately detect the position and amplitude of the breakdown point. Figure 2(d) and Figure 2(e) show a zoomed view of the position of the breakdown point in the input signal, which starts at the sample number 306 and ends at the sample number 307, and the detected position by the proposed IAF, which shows that the breakdown point is detected accurately.

The second signal was a simulation of a faulty power line, the fault starts at sample number 2052 and ends at 2053. Figure 3 shows the simulated signal and the obtained results; arranged in a similar manner to Figure 2 . The parameters of the adaptive filters, which were used in the simulation studies are given in Table 1.

However, in both simulations, the amplitude of the valley in the error signal at the position of the detected points was larger when the adaptive prediction was used. The difference between the values before the breakdown point (past) and the values at the position of the breakdown (future) produces a large prediction error. On the other hand, the difference between the extremely short-duration event in the input signal, i.e. the breakdown point, and its smoothed counterpart

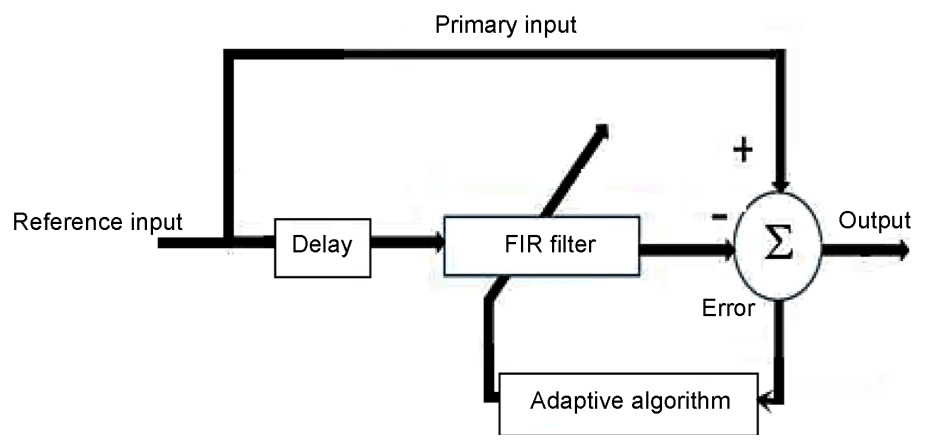

Figure 1. Adaptive prediction. 


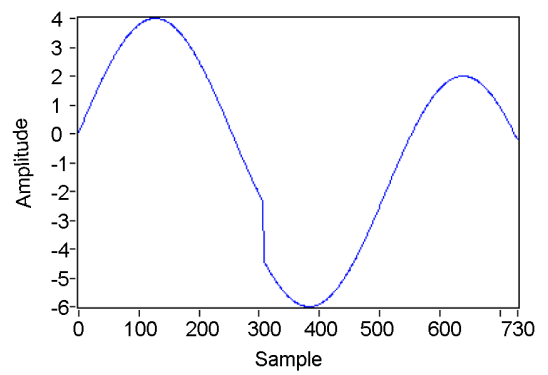

(a)

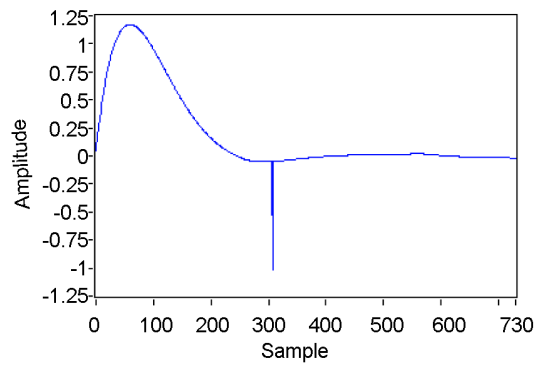

(c)

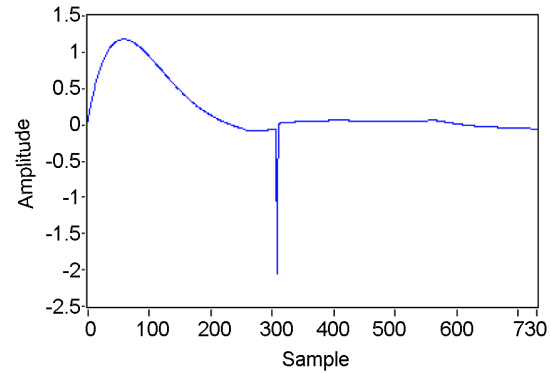

(b)

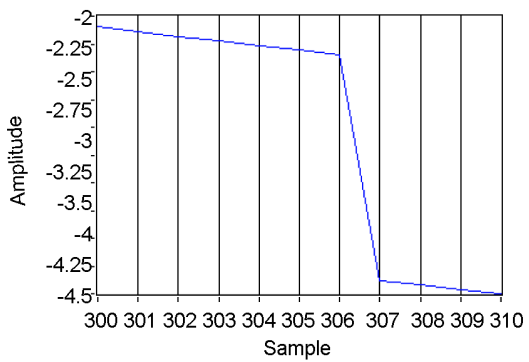

(d)

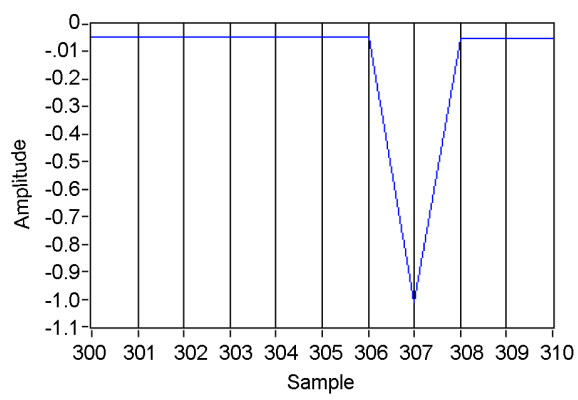

(e)

Figure 2. a) Simulated sine wave with a breakdown point; b) error signal of the adaptive linear predictor; c) error signal of the IAF; d) zoomed in view of the position of breakdown point in the input signal; e) zoomed in view of the position of the detected breakdown point in the error signal of IAF.

Table 1. Parameters of the adaptive filters used in the simulation studies.

\begin{tabular}{ccc}
\hline Signal & Filter length & Step-size \\
\hline Sine & 2 & 0.013 \\
Powerline & 2 & 0.1 \\
\hline
\end{tabular}

in the output; produces a smaller value in the error signal of the proposed filter. This results in the lower amplitude of the peak or the valley, however; it is not a limitation since the amplitude is large enough to detect.

The robustness of the proposed method in a noisy environment was investigated. A Gaussian white noise of a 0.15 standard deviation was added to the sine wave used in the first simulation, the result is given in Figure 4. The noise in the input signal resulted in a noisy error signal and caused the baseline to wander. The amplitude of the valley at the breakdown point was larger than that before adding the noise. The noisy and wandering baseline do not hinder seeking the 


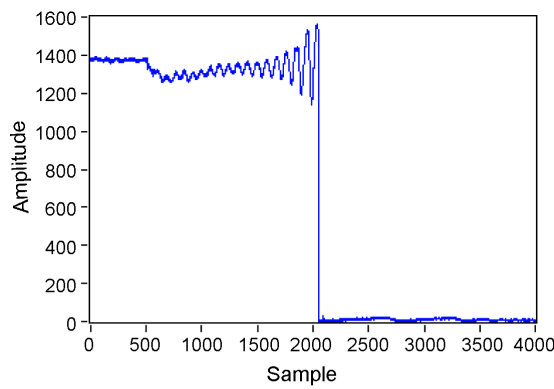

(a)

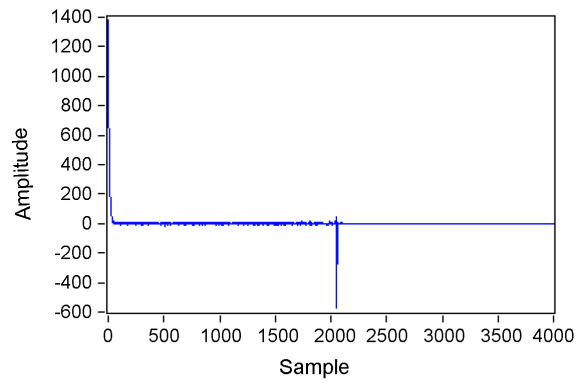

(c)

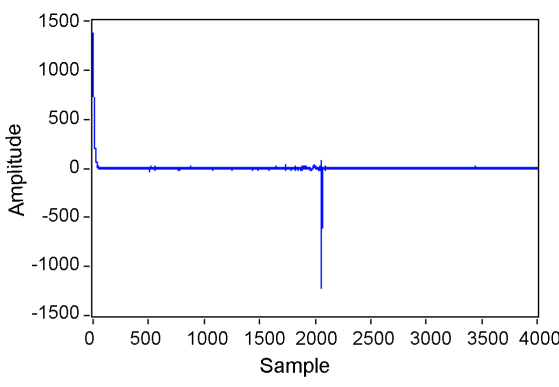

(b)

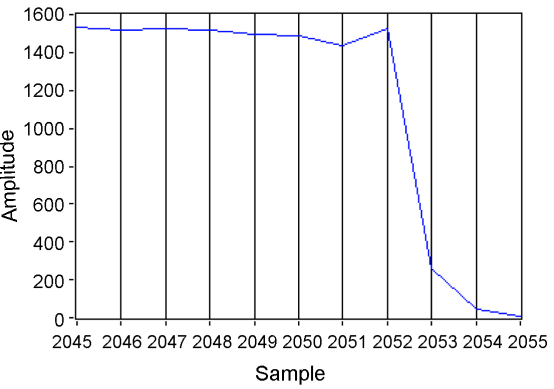

(d)

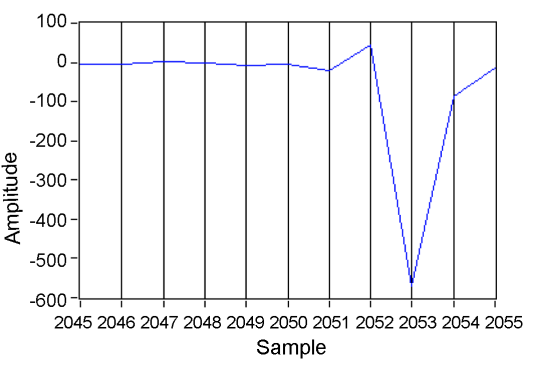

(e)

Figure 3. a) Simulated faulty powerline signal; b) error signal of the adaptive linear predictor; b) error signal of the IAF; d) zoomed in view of the position of breakdown point in the input signal; e) zoomed in view of the position of the detected breakdown point in the error signal of IAF.

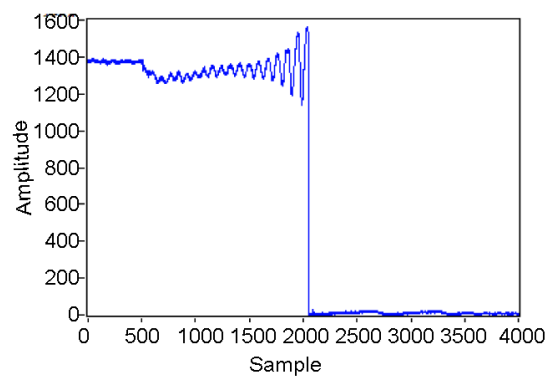

(a)

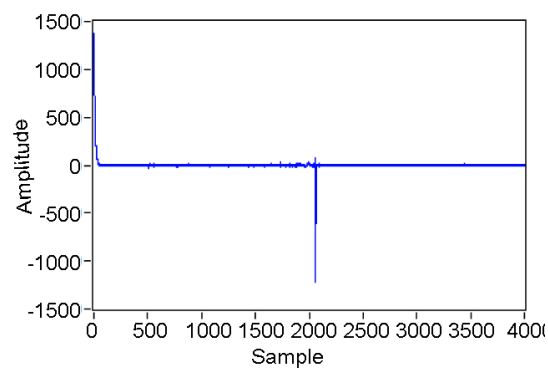

(b)

Figure 4. a) Simulated noisy sine wave; b) error signal of the proposed IAF.

onset of the breakdown point since the search starts from the valley.

\section{Experimental Results}

The experimental signals were acquired from a system being investigated to de- 
velop a spatial velocimetry method. However, the system is still under development and the signals used in this paper were from the preliminary results. In the spatial velocimetry method, the velocity of moving particles is measured using two optical fibers, the detection of a particle passage produces two signals; one when the particle enters the field of the fiber, and the other one when the particle leaves. The system under investigation produces a signal with a rapid transition (inflection point) if the particle is larger than the distance between the two fibers. The inflection point occurs at the falling shoulder in the signal depicted in Figure 5(a), and the zoomed view in Figure 5(b) shows the position of the inflection point. A digital oscilloscope was used to acquire and store the signal

The experimental results were in an agreement with the simulation results. The performances of adaptive predictor and the proposed filter were comparable. Simulation and experimental studies showed that the proposed filter can perform the function of the adaptive predictors, with the advantage of avoiding the delay in the reference input.

\section{Computational Speed}

The delay in the input to produce the filter's reference signal in the adaptive prediction introduces a delay in the computation speed of the filter. The proposed IAF proved a comparable efficiency to that of the adaptive prediction in the breakdown point detection. To demonstrate the advantage of the proposed filter, which used no delay, over the adaptive predictor; the computation times for both methods, which were implemented in a personal computer were calculated using a LabVIEW program. The results are shown in Table 2.

These results show that the proposed filter is significantly faster than the adaptive predictor, it offered a substantial decrease in the computation speed by $96.8 \%$ and $97.4 \%$ in the simulation signals, and $97.3 \%$ in the experimental signal.

This significant improvement makes the proposed system more efficient in the real-time measurements and in the systems where speed and memory matter.

\section{Conclusion}

It was shown that the performance of the proposed Identical Inputs-Adaptive Filter in the detection of the breakdown points is comparable to that of the adaptive predictor. The proposed filter requires no delay in the input signal, which significantly improves the performance and the computation speed, and

Table 2. Computation times of the adaptive linear predictor and the proposed IAF.

\begin{tabular}{ccc}
\hline \multirow{2}{*}{ Signal } & \multicolumn{2}{c}{ Computation time $(\mathrm{ms})$} \\
\cline { 2 - 3 } & Adaptive predictor & Proposed filter \\
\hline Sine & 1.9 & 0.06 \\
Powerline & 10.3 & 0.27 \\
Experimental & 0.75 & 0.02 \\
\hline
\end{tabular}




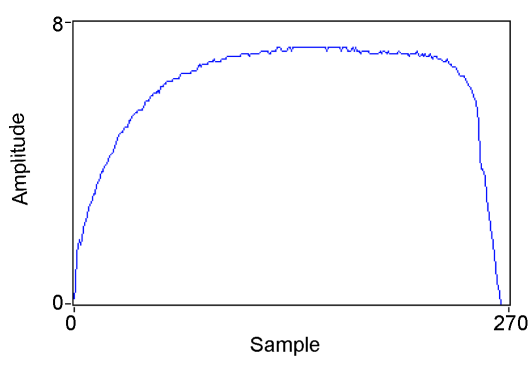

(a)

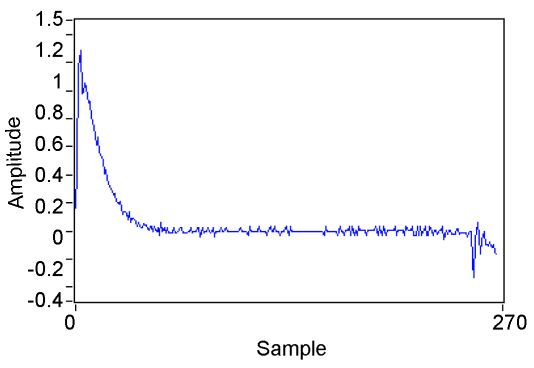

(c)

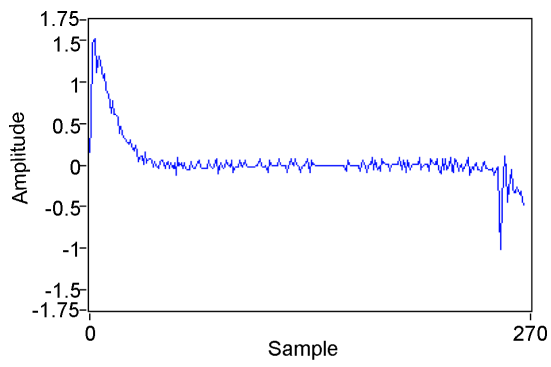

(b)

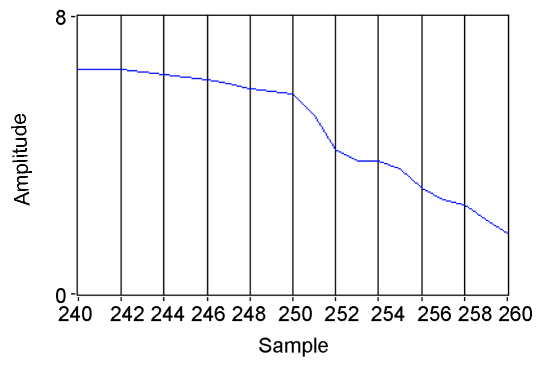

(d)

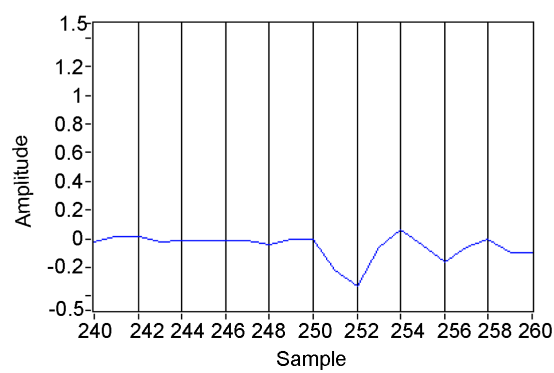

(e)

Figure 5. a) Experimental signal; b) error signal of the adaptive linear predictor; c) error signal of the IAF; d) zoomed in view of the position of breakdown point in the input signal; e) zoomed in view of the position of the detected breakdown point in the error signal of IAF.

offers an efficient alternative for the time-consuming adaptive predictor in the real-time applications.

\section{References}

[1] Mallat, S. and Hwang, W.L. (1992) Singularity Detection and Processing with Wavelets. IEEE Transaction on Information Theory, 38, 617-643. https://doi.org/10.1109/18.119727

[2] Basseville, M. and Nikoforov, I.V. (1993) Detection of Abrupt Changes-Theory and Applications. Prentice-Hall, Englewood Cliffs, NJ.

[3] Osowski, S. and Majkowski, A. (1999) Wavelet Transform Approach to the Detection of the Breaking Point of the Transient Signal in the Circuit. Proceedings of the 6 th IEEE International Conference on Electronics, Circuits and Systems, 1, 549-552.

[4] Angrisani, L., D’Apuzzo, M. and Testa, A. (1998) A Measurement Method Based on the Wavelet Transform for Power Quality Analysis. IEEE Transaction on Power Delivery, 13, 990-998. https://doi.org/10.1109/61.714415

[5] Nguyen, D.T. and Hoang, T.A. (1999) Detection of Disturbances on Electricity 
Supply Using Wavelets. Proceedings of Australian Universities Power Engineering Conference and IEAust Electric Energy Conference, Darwin, NT Australia, 1999, 184-189.

[6] Diniz, P.S.R (2008) Adaptive Filtering-Algorithms and Practical Implementation. Springer, New York.

[7] Wang, F.L. and Mechefske, C.K. (2006) Adaptive Modeling of Transient Vibration Signals. Mechanical Systems and Signal Processing, 20, 825-842. https://doi.org/10.1016/j.ymssp.2004.12.004

[8] Antunovic, M. and Cummer, S.A. (2004) Adaptive Filter for Event-Based Signal Extraction. Automatika: Journal for Control, Measurement, Electronics, Computing and Communications, 45, 129-135.

[9] Abdel-Galil, T.K., El-Saadany, E.F. and Salama, M.M.A. (2003) Power Quality Event Detection Using Adaline. Electric Power Systems Research, 64, 137-144. https://doi.org/10.1016/S0378-7796(02)00173-6

[10] Sayed, A.H. (2008) Adaptive Filters. John Wiley \& sons Inc, Hoboken, New Jersey, USA.

Submit or recommend next manuscript to SCIRP and we will provide best service for you:

Accepting pre-submission inquiries through Email, Facebook, LinkedIn, Twitter, etc. A wide selection of journals (inclusive of 9 subjects, more than 200 journals) Providing 24-hour high-quality service User-friendly online submission system Fair and swift peer-review system Efficient typesetting and proofreading procedure Display of the result of downloads and visits, as well as the number of cited articles Maximum dissemination of your research work

Submit your manuscript at: http://papersubmission.scirp.org/ Or contactwjet@scirp.org 